\title{
Testing alone is insufficient
}

\author{
Rahul Deb ${ }^{1} \cdot$ Mallesh Pai ${ }^{2} \cdot$ Akhil Vohra $^{3} \cdot$ Rakesh Vohra ${ }^{4}$
}

Received: 18 October 2021 / Accepted: 22 January 2022 / Published online: 1 March 2022

(c) The Author(s), under exclusive licence to Springer-Verlag GmbH Germany, part of Springer Nature 2022

\begin{abstract}
The fear of contracting a serious illness caused by a contagious disease limits economic activity even after reopening. Widespread testing alone will not alleviate this problem. We argue that targeted testing in concert with targeted transfers is essential. We propose a model with these features to determine where agents should be tested and how they should be incentivized. Agents with a low wage, a high risk of infection, and who bear a large cost of falling ill should be tested at work. When testing is very costly, agents with high wages and low expected costs associated with falling ill should be tested at home.
\end{abstract}

Keywords Epidemics · Mechanism design · COVID-19 · Health policy

JEL Classification D04 · D62 · I18

We would especially like to thank Olivier Gossner for valuable comments and suggestions. We also thank three anonymous referees for their comments and Huseyin Yildirim for handling the manuscript. Penultimate author was supported in part by ERC Grant \#757229 under the European Union's Horizon 2020 research and innovation program. The last author gratefully acknowledges the support of the Rockefeller Foundation through Grant 2017PRE301.

\section{Rakesh Vohra}

rvohra@seas.upenn.edu

Rahul Deb

rahul.deb@utoronto.ca

Mallesh Pai

Mallesh.Pai@rice.edu

Akhil Vohra

av615@cam.ac.uk

1 Department of Economics, University of Toronto, Toronto, ON, Canada

2 Department of Economics, Rice University, Houston, TX, USA

3 Department of Economics, University of Cambridge, Cambridge, UK

4 Department of Economics, University of Pennsylvania, Philadelphia, PA, USA 


\section{Introduction}

It is a truth universally acknowledged that an economy needing to recover from a pandemic-induced coma must be in need of inexpensive and widespread testing.

We argue that this by itself is insufficient. Suppose that to reduce the spread of the virus, we must ensure that the fraction of infected individuals who are out and about is below some threshold. With unlimited testing, we could test each and every person, but what will they do with the information gleaned? Why should we expect someone who has tested positive for the virus to stay home and someone who has tested negative to go to work? If the first receives no compensation for staying home, she may choose to leave for work. The second, anticipating that infected individuals will show up for work, may choose to stay home. ${ }^{1}$ As a result, the fraction of the population out and about will have an infection rate exceeding that in the population at large. Even if the prevalence of the infection is low, most agents may choose to stay home.

This paper makes two points. First, in the absence of a cure or effective vaccine, any plan to restart the economy must combine testing with incentives. It is not enough to reward individuals for getting tested, as proposed by Levitt et al. (2020). One must shape behavior: compensate the infected for staying home and incentivize the uninfected to go out. Testing allows us to identify who should be the beneficiary of these transfers. Combining targeted testing with well-designed incentives results in better outcomes than either by themselves. Our second point is that where one tests also matters. There is a crucial difference between testing at work and testing 'at home'. Testing at home should be interpreted as a way to test an individual without increasing exposure to others. An infected person who leaves home to be tested at work poses an infection risk to others who choose to go outside. ${ }^{2}$ The precise mix of home and work testing depends, as we argue, on the level of infection risk agents are prepared to accept. The more willing agents are to go out, the more one should focus on random testing at home. If the reverse, one should focus on testing at work. If there were no delay between test and result, the distinction between home and work testing would be meaningless. One could ask an agent to test at home and report to work if they receive a negative result. The distinction matters as long as there is a substantial delay between taking the test and receiving a result (which is often the case in reality). With regards to COVID-19, not only do PCR test results arrive after a 2-5 day delay but there is an incubation period for the virus: a positive test result may not show up until 5-8 days after infection has occurred.

Our claims are based on a simple model where individuals choose whether to stay in or go out and work. They decide given their subjective evaluation of costs and benefits. A planner has access to costly testing and can influence incentives by selecting rates of testing of those who stay at home and those who work, and contingent transfers to agents based on their choice and their test outcomes. We consider two scenarios:

\footnotetext{
1 A June 17th, 2020, survey conducted by the consulting firm Korn Ferry of 1,044 American professional workers found that half were reluctant to go back to the office citing health concerns.

2 More generally, the reader might imagine a testing authority who monitors those activities that create transmission opportunities and conducts tests with a likelihood that depends on activity levels.
} 
1. A random subset of those who have elected to stay home are tested. If they test positive or are untested, they receive a subsidy to remain at home. If they test negative, they receive no subsidy.

2. A random subset of those who have elected to go out are tested. Those who are untested or test negative receive their wage and possibly a premium. Those who test positive receive no wage.

Our main finding is that when fear of infection is high, agents who are engaged in low-wage work with a high expected cost of falling ill should be tested at work only. On the other hand, those with high wages, low risk of being infected, and low cost of falling ill should generally be tested at home.

The intuition is as follows. The optimal test-at-home policy involves a wage premium to those that work and a transfer to those who test positive at home. If the wage is small relative to the cost associated with becoming sick, those likely to be uninfected have a strong incentive to stay home. To incentivize the healthier individuals to work, a test-at-home policy would require a substantial wage premium that would encourage all types, including those likely to be infected, to work. An attractive transfer contingent on staying home would then be needed to dissuade these types.

On the other hand, the optimal test-at-work policy involves a transfer to those at work who test negative and a transfer to those staying at home that is inversely proportional to the probability of being tested at work. The presence of a conditional transfer at work deters those likely to be infected, as they would receive nothing if the test result were positive. As a consequence, the home transfer can be lowered. When the wage is relatively high, and the cost of infection is low, testing at work is a deterrent if one can test at a high rate. When testing is sufficiently costly, such a test rate is infeasible, so testing at home is cheaper.

The idea is straightforward. In the absence of widespread testing to distinguish between the infected and those who are not, we must rely on individuals to sort themselves. They are in the best position to determine the likelihood they are infected (e.g. based on private information about exposures, how rigorously they have been distancing etc.). Properly tailored transfers give them the incentive to do so. By tailoring the transfers and the testing policy to individual circumstances, one achieves the same goal at an even lower cost.

The next section summarizes related literature. Section 3 describes the model and Sect. 4 contains the main findings. We reserve the discussion of our assumptions, as well as how the scheme of targeted testing and transfers might be implemented, to Sect. 5.

\section{Related literature}

Our paper adds to the rapidly growing literature on the economic aspects of pandemics inspired by COVID-19. ${ }^{3}$ The issues considered are not specific to COVID-19 but any

\footnotetext{
3 There is a new journal dedicated to economic analysis related to COVID-19: https://cepr.org/content/ covid-economics-vetted-and-real-time-papers- 0 .
} 
contagious pandemic that is severe enough to cause agents to dramatically reduce economic activity, either individually or in concert (i.e. lockdowns).

Much of the burgeoning literature centers on examining disease spread and effectiveness of lockdowns via an SIR model (Atkenson 2020; Acemoglu et al. 2020; Engle et al. 2021). These papers modify and calibrate SIR models to account for heterogeneous populations, targeted lockdowns, and social distancing measures. Other papers examine the effectiveness of physical distancing and containment policies in reducing contagion, for example, Akbarpour et al. (2020); Alvarez et al. (2020); Bueno de Mesquita and Shadmehr (2020) and Kruse and Strack (2020). A related stream of papers assesses the macroeconomic impact of infection and containment policies (Eichenbaum et al. 2020; Favero et al. 2020; Glover et al. 2020). Three recent theoretical papers (McAdams 2020; Dasaratha 2020; Carnehl et al. 2021) incorporate behavioral responses but not private information into the SIR model; all agents share the same common knowledge belief about the chance they are infected. These papers do not address the issue of how to encourage economic activity under the threat of infection.

Beginning with Dorfman (1943) and continuing with Du and Hwang (1992), there is an extensive literature on how to use limited or costly testing to extract as much information as possible. The use of such methods in the context of COVID-19 has been discussed by Gollier (2020), Gollier and Gossner (2020), Lipnowski et al. (2021), Ely et al. (2021), and Bobkova et al. (2021). This stream of work is not focused on how the information gleaned from testing is best used.

The literature that explores how testing will be used views it as a device to decide which agents should be quarantined to reduce transmission rates. Quarantining those who test positive for infection counters the externality imposed by the infected. The focus is on how to trade-off the amount of testing and quarantining with the level of economic activity. Examples are Alvarez et al. (2020), Eichenbaum et al. (2020), and Farboodi et al. (2020). They differ in whether agents know their health status, the dynamics of infection (e.g. are those who recover immune or are they still susceptible), and the planner's objective function (aggregate output, labor supply or consumption). However, it is assumed that an agent released from quarantine will choose to go to work. This paper is concerned with providing the incentives for such a selection. On a related front, there is a large literature motivated by earlier pandemics on the allocation of scarce resources to control spread, to test, or to meet patient demand (Preciado et al. 2014; Armbruster and Brandeau 2007; Bienstock and Zenteno 2015).

Lastly, there is an extensive theoretical literature on mechanism design with verification (Ben-Porath et al. 2014; Li 2020). These models do not incorporate externalities nor distinguish between types of testing and verification.

None of the prior work we are aware of examines the use of targeted testing and targeted transfers to compensate for limited testing, segment the population, and induce a specific infection rate in the workforce. The Safra Center's pandemic report recommends support for those who test positive and are placed in isolation. ${ }^{4}$ However, this is in the context of a regime with large scale testing. We point out that if testing is

\footnotetext{
4 https://ethics.harvard.edu/files/center-for-ethics/files/roadmaptopandemicresilience_updated_4.20.20_ 0.pdf.
} 
limited, one should be prepared to support some who have not been tested. We also propose incentives to encourage people to go to work.

\section{Model}

Assume a unit mass of agents of which a fraction $\pi_{I}$ can transmit the virus. For brevity, call this group infected. Conditional on being infected, the probability of displaying symptoms is $\pi_{S}$. Therefore, we allow for the possibility that some agents may be asymptomatic carriers.

We assume each agent has a belief about the likelihood that they carry the virus and that the belief is calibrated (i.e. among all the agents who believe they are infected with probability $b$, a fraction $b$ are actually infected). They form this belief based on prior activities, whom they have come into contact with, and what symptoms they may have. Every symptomatic agent understands that they are infected. Beliefs are drawn from a distribution $G$ over $[0,1]$ that satisfies the following two conditions:

$$
\begin{aligned}
& \int_{0}^{1} b \mathrm{~d} G(b)=\pi_{I}, \\
& G(1)-\lim _{b \uparrow 1} G(b)=1-\lim _{b \uparrow 1} G(b)=\pi_{S} \pi_{I} .
\end{aligned}
$$

The former condition requires that the average belief in the population is correct - this is implied by our assumption that beliefs are calibrated. The latter condition asserts that the mass of agents who display symptoms correctly believe that they are infected: there is an atom at 1 , and it is comprised of the symptomatic agents. Note that an individual with belief $b<1$ is either not infected or infected with no symptoms.

The decision that each individual with belief $b \in[0,1]$ must make is whether to stay indoors or go outside. When outdoors, an individual works and engages in other activities that generate economic benefits, such as going to bars or the cinema. Our focus is not on how they choose to allocate their time between these activities, only on whether they decide to go outdoors or not. What an agent chooses depends on the chance they are infected and the chance of being infected if they go outside.

Denote by $l(b) \in[0,1]$ the probability that an agent with belief $b$ chooses to go out. Thus, the fraction of agents out of their homes is $\int_{0}^{1} l(b) \mathrm{d} G(b)$, and the fraction of infected agents among those outside their homes is

$$
z=\frac{\int_{0}^{1} b l(b) \mathrm{d} G(b)}{\int_{0}^{1} l(b) \mathrm{d} G(b)}
$$

If $z$ is the initial proportion of infected individuals outside, the probability an individual without the virus becomes infected (over some relevant period) is $f(z)$, where $f$ is a continuous function. The function $f$ does not depend on the size of the population that goes out to work, and in Sect. 5, we show that this is without loss for the 
social planner. ${ }^{5}$ The expected personal cost conditional on being infected is $c>0$. Included in $c$ is the probability $\pi_{S}$ of becoming symptomatic. In Sect. 5.2, we discuss the consequences of allowing heterogeneous costs. Lastly, each agent has a common wage $w$. For our purposes, this is also without loss. ${ }^{6}$

The task of the social planner is to choose carrots, sticks, and a testing policy to achieve a given response among the population. For the first part of this paper, we focus on the following goal for the planner: achieve a target rate of infection among the working population of $z^{*}<\pi_{I}{ }^{7}$ where the agents who go out are those with beliefs $b \leq b^{*}$ (i.e. $l(b)=1$ if $b \leq b^{*}, l(b)=0$ otherwise). ${ }^{8}$ The question we study is what is the minimum cost way of inducing this? In Sect. 5.1 we study a general planner's problem and show that it reduces to the problem of selecting this target infection rate $z^{*}$, or equivalently, the interval of agents with belief in $\left[0, b^{*}\right]$ to go out.

At its disposal, the planner has the following instruments available:

1. Let $r_{H}$ and $r_{W} \in[0,1]$ be the probabilities of testing an individual conditional on whether she is inside $(H)$ or outside $(W)$. We will often call the first 'staying home' and the second 'work'?

2. The planner can levy transfers $t_{d}^{o}$ contingent on an agent's in/out decision $d \in$ $\{H, W\}$ and test outcome $o \in\{\neg,-,+\}$ (respectively, not tested, tested negative and tested positive).

For the transfers to be politically palatable, we assume two restrictions. Firstly, we assume that all transfers are non-negative-this captures the usual limited liability constraint. Secondly, agents who go to work and are either not tested or test negative receive, at minimum, their wage. Formally:

$$
t_{d}^{o} \geq 0, t_{W}^{\neg}, t_{W}^{-} \geq w .
$$

By only requiring that $t_{W}^{+} \geq 0$, we assume that agents who test positive can, for example, be furloughed without a wage.

The difference between testing at home and work is not cosmetic. Infected agents who stay in their homes and are tested there do not pose an infection risk to those who choose to go outside. Testing at home should be interpreted to cover all methods to test an individual without increasing exposure to others. Conversely, an infected agent who chooses to go outside raises the risk of infection to others outside, regardless of whether she will subsequently be tested 'at work'. Even if agents are sent home after

\footnotetext{
5 This is not without loss for an individual because the interaction rate with others could vary by their choice of activity. It suggests that transfers should depend on industries.

6 Wages are observable to the planner, and so policies can be conditioned on agents' wages.

7 One could just as well look at $z^{*}>\pi_{I}$, which would involve incentivizing the healthy to stay home. Our analysis would apply with the appropriate inequalities flipped - we focus on the case of $z^{*}<\pi_{I}$ because the social planner objective of incentivizing the healthy to work and the sick to stay home is subjectively more natural. However, a policy designed to keep the healthy at home would be natural when it is likely that most of the population is already infected.

8 Therefore, $b^{*}$ and $z^{*}$ are linked by (1).

9 Testing at home is already feasible given current technology. For example, we have access to phone applications that verify the results of self-administered tests.
} 
having tested positive, they have raised the risk of infection to others because they engaged in activities like going to a restaurant or using mass transit.

To summarize, the timing of the model is as follows:

1. The planner decides on testing probabilities and transfers.

2. Each individual forms a belief about their infection status and decides whether to stay home or go to work.

3. Tests are conducted, and transfers are awarded. Some of the healthy individuals who go out are infected.

4. Payoffs are realized.

Given any policy $P=\left(r_{d}, t_{d}^{o}: d \in\{H, W\}, o \in\{+,-, \neg\}\right)$, let $V(b, l, z, P)$ denote the value to an agent with belief $b$ who works with probability $l$. Note that:

$$
\begin{aligned}
& V(b, 0, z, P)=r_{H}\left(b t_{H}^{+}+(1-b) t_{H}^{-}\right)+\left(1-r_{H}\right) t_{H}^{\neg} \\
& V(b, 1, z, P)=r_{W}\left(b t_{W}^{+}+(1-b) t_{W}^{-}\right)+\left(1-r_{W}\right) t_{W}^{\neg}(1-b) c f(z) \\
& V(b, l, z, P)=V(b, 0, z, P)+l(V(b, 1, z, P)-V(b, 0, z, P)) .
\end{aligned}
$$

In words, given a policy $P$, an agent who stays at home (goes to work) expects to be tested with probability $r_{H}\left(r_{W}\right)$ and receive transfers based on whether they got tested and the outcome of the test. Their expected value of this depends on their belief $b$ that they are infected. Regardless of whether they go to work or stay at home, an agent may develop symptoms and suffer the cost of infection. Additionally, an agent who goes to work faces the risk of getting infected at work-this depends also on the fraction of agents at work who are infected. Under policy $P$, an agent with belief $b$ that expects the infection rate among those outside to be $z$ will go outside if the value from working is greater than from staying at home. The agent will stay at home otherwise.

Definition 3.1 Policy $P$ is consistent with and induces infection rate $z$ if there exists a corresponding "going-out" function $l(\cdot)$ such that:

1. $l(b)>0 \Longrightarrow V(b, 1, z, P)-V(b, 0, z, P) \geq 0$,

2. $l(b)<1 \Longrightarrow V(b, 1, z, P)-V(b, 0, z, P) \leq 0$,

3. and $z=\frac{\int_{0}^{1} b l(b) d G(b)}{\int_{0}^{1} l(b) d G(b)}$.

The expression $V(b, 1, z, P)-V(b, 0, z, P)$ is linear in $b$. If the partial derivative of $V(b, 1, z, P)-V(b, 0, z, P)$ with respect to $b$ is strictly positive, the set of agents who go to work will be agents with beliefs in some set $\left[b^{*}, 1\right]$. Thus, for the target infection rate $z^{*}$ to be less than $\pi_{I}$, it must be that $\frac{\partial V\left(b, 1, z^{*}, P\right)-V\left(b, 0, z^{*}, P\right)}{\partial b}$ is non-positive:

$$
c f\left(z^{*}\right)+r_{H}\left(t_{H}^{-}-t_{H}^{+}\right)+r_{W}\left(t_{W}^{+}-t_{W}^{-}\right) \leq 0 .
$$

As we said earlier, we focus on policies such that the set of agents that are out is the subset with beliefs in $\left[0, b^{*}\right]$ for some $b^{*}$. Consistency of the policy $P$ with infection 
rate $z^{*}$ therefore reduces to $\int_{0}^{b^{*}} b \mathrm{~d} G(b)=G\left(b^{*}\right) z^{*}$. Further, $b^{*}$ must be such that $V\left(b^{*}, 1, z^{*}, P\right)-V\left(b^{*}, 0, z^{*}, P\right)=0$ or equivalently that

$$
b^{*}=\frac{c f\left(z^{*}\right)+\left(1-r_{H}\right) t_{H}^{-}-\left(1-r_{W}\right) t_{W}^{-}-r_{W} t_{W}^{-}+r_{H} t_{H}^{-}}{c f\left(z^{*}\right)+r_{H}\left(t_{H}^{-}-t_{H}^{+}\right)+r_{W}\left(t_{W}^{+}-t_{W}^{-}\right)} .
$$

The cost of such a policy, net of wages, is

$$
\begin{aligned}
& M\left(\left(1-G\left(b^{*}\right)\right) r_{H}+G\left(b^{*}\right) r_{W}\right)+r_{H}\left[\left(\pi_{I}-z^{*} G\left(b^{*}\right)\right)\left(t_{H}^{+}-t_{H}^{-}\right)+\left(1-G\left(b^{*}\right)\right) t_{H}^{-}\right] \\
& \quad+r_{W}\left[z^{*} G\left(b^{*}\right)\left(t_{W}^{+}-t_{W}^{-}\right)+G\left(b^{*}\right) t_{W}^{-}-\left(1-z^{*}\right) G\left(b^{*}\right) w\right] \\
& \quad+\left(t_{W}^{\neg}-w\right)\left(1-r_{W}\right) G\left(b^{*}\right)+t_{H}^{\neg}\left(1-r_{H}\right)\left(1-G\left(b^{*}\right)\right) .
\end{aligned}
$$

Here, $M:[0,1] \rightarrow \mathbb{R}_{+}$is an increasing, convex function that captures the cost of testing a given fraction of the population.

In what follows, we focus on comparing two different environments: testing only at home and testing only at work. Therefore, the problem for a planner considering testing only at home $\left(r_{W}=0\right)$ is:

$$
\begin{aligned}
\inf _{P}\left\{M\left(\left(1-G\left(b^{*}\right)\right) r_{H}\right)+r_{H}\left[\left(\pi_{I}-z^{*} G\left(b^{*}\right)\left(t_{H}^{+}-t_{H}^{-}\right)+\left(1-G\left(b^{*}\right)\right) t_{H}^{-}\right]\right.\right. \\
\left.\quad+\left(t_{W}^{\neg}-w\right) G\left(b^{*}\right)+t_{H}^{\neg}\left(1-r_{H}\right)\left(1-G\left(b^{*}\right)\right)\right\}, \\
\quad \text { s.t. (2), (3), (4). }
\end{aligned}
$$

Similarly, the problem for a planner considering testing only at work $\left(r_{H}=0\right)$ is:

$$
\begin{aligned}
\inf _{P}\left\{M\left(G\left(b^{*}\right) r_{W}\right)+r_{W}\left[z^{*} G\left(b^{*}\right)\left(t_{W}^{+}-t_{W}^{-}\right)+G\left(b^{*}\right) t_{W}^{-}-\left(1-z^{*}\right) G\left(b^{*}\right) w\right]\right. \\
\left.\quad+\left(t_{W}^{\neg}-w\right)\left(1-r_{W}\right) G\left(b^{*}\right)+t_{H}^{-}\left(1-G\left(b^{*}\right)\right)\right\},
\end{aligned}
$$

s.t. (2), (3), (4).

We write an infimum instead of a minimum because the optimal policy may not be (and in some cases is not) well defined - it may involve unbounded payments with infinitesimal testing probabilities. Since the cost function is non-negative, the infimum will exist. Thus, standard arguments tell us that there are well defined policies that get arbitrarily close to the infimum. ${ }^{10}$

\footnotetext{
10 Note that we do not explicitly impose a "budget balance" constraint because the planner could simply cover the costs via a lump-sum tax on all individuals.
} 


\section{Incentivizing a target infection rate}

\subsection{Testing with no transfers}

To understand the value of transfers in this setting, consider first a benchmark in which only testing is available but no additional transfers are permitted (agents who go to work receive their wage, while agents who stay home receive nothing). We observe below that it is impossible to induce an infection rate in the working population below the infection rate in the overall population.

Observation 4.1 If there are no transfers, i.e. $t_{H}^{+}=t_{H}^{-}=t_{H}^{\neg}=0$ and $t_{W}^{+}=t_{W}^{-}=$ $t_{W}^{\neg}=w$, it is impossible to induce an infection rate of $z^{*}<\pi_{I}$ in the working population.

To see this, observe that in the absence of transfers, inequality (3) reduces to $c f\left(z^{*}\right)<0$, which cannot be as the expected cost of infection is positive. Intuitively, without transfers, it is impossible to deter the individuals likely to be infected from going to work. Therefore, we cannot induce an infection rate in the working population that is lower than the base rate without subsidies or penalties. Hence, testing alone, even if plentiful, is insufficient. ${ }^{11}$

\subsection{Work testing only}

Consider a policy of only testing at work, so $r_{H}=0$. We think of this as the employer testing their employees with probability $r_{W}$. In this case, $(3,4)$ simplify to

$$
\begin{aligned}
& c f\left(z^{*}\right)+r_{W}\left(t_{W}^{+}-t_{W}^{-}\right) \leq 0, \\
& b^{*}=\frac{c f\left(z^{*}\right)+t_{H}^{\neg}-\left(1-r_{W}\right) t_{W}^{-}-r_{W} t_{W}^{-}}{c f\left(z^{*}\right)+r_{W}\left(t_{W}^{+}-t_{W}^{-}\right)} .
\end{aligned}
$$

A simple argument delivers that in any minimum-cost policy, any agent testing positive is furloughed-intuitively, this optimally disincentivizes agents who believe they are likely to be sick from going to work.

Lemma 4.2 In any minimum-cost policy, we must have that $t_{W}^{+}=0$.

This simplifies the constraints (3), (4) further to

$$
\begin{aligned}
& c f\left(z^{*}\right)-r_{W} t_{W}^{-} \leq 0, \\
& b^{*}=\frac{c f\left(z^{*}\right)+t_{H}^{\neg}-\left(1-r_{W}\right) t_{W}^{\neg}-r_{W} t_{W}^{-}}{c f\left(z^{*}\right)-r_{W} t_{W}^{-}} .
\end{aligned}
$$

11 Proposition 13B.2 in Mas-Colell et al. (1995) tells us that levying transfers in the absence of testing information is also unhelpful. They consider an adverse selection model of hiring. A planner with no access to information is limited to using lump-sum transfers and cannot implement the full information Pareto optimal outcome. 
Fixing the testing probability $r_{W}$, we have two possible regimes to consider. The first case, $c f\left(z^{*}\right)-r_{W} w<0$, corresponds to a setting where at an expected infection rate of $z^{*}$, the wage is sufficiently large so that with no additional incentives beyond wage deduction, too many individuals likely to be infected will choose to work. This will crowd out those with a low likelihood of infection. The second case is $c f\left(z^{*}\right)-r_{W} w>$ 0 , which corresponds to the opposite setting: too few people choose to go out.

Proposition 4.3 Suppose testing is only conducted at work $\left(r_{H}=0\right)$. Conditional on the testing probability $r_{W}$, the minimum-cost transfers are as follows:

1. If $c f\left(z^{*}\right)-r_{W} w<0$, the optimal transfer policy has $t_{H}^{\neg}>0, t_{W}^{-}=w$, and $t_{W}^{\neg}=w:$ a transfer to those that stay home, and no transfers in excess of the wage to those that go to work and either test negative or are untested.

2. If $c f\left(z^{*}\right)-r_{W} w>0$, the optimal transfer policy has $t_{H}^{\overrightarrow{ }}>0, t_{W}^{-}>w$, and $t_{W}^{\neg}=w:$ a transfer to those that stay home, and a transfer in excess of the wage to those that work and test negative. Those untested at work receive only their wage.

The value of the minimum-cost policy as a function of the test-at-work probability, $r_{W}$, is continuous in $r_{W}$. Finding the optimal $r_{W}$ amounts to optimizing a continuous function over a compact set. Therefore, when constrained to testing at work, an optimal testing probability exists. Moreover, it can be easily derived if we impose sufficient structure on the cost of testing $M$ and the belief distribution $G$.

\subsection{Home testing only}

Now suppose there is no testing at work (so $r_{W}=0$ ) but testing is instead conducted at home. The expression for $b^{*}$ in (4) simplifies to:

$$
b^{*}=\frac{c f\left(z^{*}\right)+\left(1-r_{H}\right) t_{H}^{\neg}-t_{W}^{\neg}+r_{H} t_{H}^{-}}{c f\left(z^{*}\right)+r_{H}\left(t_{H}^{-}-t_{H}^{+}\right)} .
$$

An analogous argument to the previous section shows that there will be no transfers to agents who stay at home and test negative. This is summarized in the following lemma.

Lemma 4.4 In any minimum-cost policy, we must have that $t_{H}^{-}=0$.

Once again, this simplifies the constraints faced by the principal and $(3,4)$ become:

$$
\begin{aligned}
& c f\left(z^{*}\right)-r_{H} t_{H}^{+} \leq 0, \\
& b^{*}=\frac{c f\left(z^{*}\right)+\left(1-r_{H}\right) t_{H}^{\neg}-t_{W}^{\neg}}{c f\left(z^{*}\right)-r_{H} t_{H}^{+}} .
\end{aligned}
$$

Proposition 4.5 Suppose testing is only conducted at home $\left(r_{W}=0\right)$. Conditional on the testing probability $r_{H}$, the minimum-cost transfers are as follows: 
1. If cf $\left(z^{*}\right)-w>0$, the optimal transfer policy has $t_{H}^{\neg}=0, t_{H}^{+}>0$, and $t_{W}^{\neg}>w$ : a transfer to those who stay home and test positive as well as a transfer in excess of the wage to those who go to work.

2. If cf $\left(z^{*}\right)-w<0$, the optimal transfer policy has $t_{H}^{\neg}>0, t_{H}^{+}>0$, and $t_{W}^{\neg}=w$ : a transfer to those that stay home and either test positive or do not get tested, and no transfer in excess of the wage to those who go to work.

While the details of the scheme are computed in Appendix A.1, two elements of the optimal test-at-home policy are important to highlight:

1. Work transfers in excess of the wage are independent of the test-at-home probability $r_{H}$.

2. In either scenario, the transfer to those that stay at home and test positive is equal to $\frac{c f\left(z^{*}\right)}{r_{H}}$. The magnitude of the transfer conditional on testing positive is inversely proportional to the testing probability.

Given this policy structure, the test-at-home probability only affects the at-home transfers. However, because the at-home transfers scale with $\frac{1}{r_{H}}$, our proof shows that the total cost of the transfers (total cost less the cost of testing) is independent of the test-at-home probability $r_{H}$. Thus:

Proposition 4.6 The value of the minimum cost test-at-home policy as a function of $r_{H}$ declines as $r_{H} \rightarrow 0$. However, the stay-at-home transfer conditional on testing positive scales with $\frac{1}{r_{H}}$, that is, $t_{H}^{+}=\frac{c f\left(z^{*}\right)}{r_{H}}$.

Hence, in the absence of an upper bound on transfers, a minimum cost test-at-home policy does not exist. The infimum can be computed by taking the limit as $r_{H} \rightarrow 0$, see Appendix A.1 for details. In reality, transfers would be bounded, so $r_{H}$ would be lowered to the point where the stay-at-home transfer meets that bound.

\subsection{Home testing vs. work testing}

When is testing at work more cost-efficient than testing at home? This is an especially important question when people are fearful of returning to work. In the context of our model, this is when $c f\left(z^{*}\right)-w>0$, i.e., the cost of getting infected exceeds the worker's wage. In this case, workers that believe they are uninfected will not want to go to work. Such was the case in, for example, Texas and Georgia in 2020: even though COVID-19 lockdowns had been lifted, people were still fearful of infection and refused to go out. ${ }^{12}$

Proposition 4.7 Suppose c $f\left(z^{*}\right)-w>0$ :

1. If $\pi_{I} \geq \frac{w}{c f\left(z^{*}\right)}$, then the minimum-cost test-at-work policy that induces infection rate $z^{*}$ is less costly than any test-at-home policy that induces infection rate $z^{*}$.

2. If $\pi_{I}<\frac{w}{c f\left(z^{*}\right)}$ and testing is sufficiently costly, there is a test-at-home policy that induces infection rate $z^{*}$ and is less costly than the minimum-cost test-at-work policy that induces infection rate $z^{*}$.

\footnotetext{
12 https://www.nbcnews.com/news/us-news/some-texas-cities-nervous-governor-reopens-state-everybody -scared-n1198691.
} 
If the wage is small relative to the cost of being ill $\left(\frac{w}{c f\left(z^{*}\right)}<\pi_{I}\right)$, it is more costeffective to use a test-at-work policy. To see why, notice that in this setting, those likely to be uninfected have a strong incentive to stay home. To incentivize healthier individuals to work, a test-at-home policy would require a substantial wage premium. Such a premium would encourage all types, including those likely to be infected, to work. Hence, an attractive transfer on testing positive is needed to keep these types indoors. However, a test-at-work policy deters those likely to be infected, as they receive nothing if their test result is positive. Thus, for jobs where the wage is low, and there is high interaction amongst individuals, testing should be conducted at work. In these industries, workers who test negative will be subsidized. Individuals remaining at home will also receive a payment.

Analogously, if the wage is large relative to the cost of being ill $\left(\frac{w}{c f\left(z^{*}\right)}>\pi_{I}\right)$, those likely to be uninfected have a strong incentive to stay home. When the wage is relatively high, and the cost of infection is low, testing at work serves as a deterrent if one can test at a high rate. Thus, when testing is sufficiently costly, such a test rate is infeasible, and so a test-at-home policy should be used.

In this analysis, we compared two policies: only testing at home versus only testing at work. It may be more cost-effective to deploy a mixture. It is possible to derive such a general characterization when more structure is imposed on the functions $M$ and $G$ in the planner's objective. We feel that the particular form of this mixed testing policy (which will depend finely on the details of the environment) is orthogonal to our main point, so we omit it for brevity.

\section{Discussion and conclusion}

Testing is important insofar as it provides an accurate estimate of the infection rate in the population. However, test results alone do not give people the incentive to engage in economic activity. A perceived high risk of infection will dampen economic activity.

In this paper, we propose that a policy of targeted testing in combination with targeted transfers is necessary to control the infection rate in the workforce and thus incentivize the "right" people to go to work. This paper provides a baseline model for thinking through the trade-offs involved. Whom should we target for testing, and where should we test? Our model highlights that the answers to this question depend on the wage earned and the expected cost borne of being infected.

Throughout the paper, we have deliberately chosen stark/ simple assumptions so as to cleanly describe the tradeoffs involved. In what follows, we discuss these in slightly more detail. Section 5.1 revisits the supposition that the planner only wishes to control the infection rate among the working population and shows it as a consequence of a general social planner's problem. Section 5.2 discusses some of the other assumptions made in the paper. Section 5.3 discusses the logistics of implementing such policies. 


\subsection{Microfoundation}

Here we state and formulate a general social planner's problem and show why its solution reduces to a choice of infection rate among the working population.

Suppose society enjoys benefit $\phi(\cdot)$ as a function of the fraction of agents who work and suffers a cost $\kappa(\cdot, \cdot)$ as a function of the infection rate in the working population and the size of the workforce. Recall that, given a function $l(b)$, which expresses the probability that an agent with belief $b$ chooses to go out, the proportion of infected individuals outside is $z=\frac{\int_{0}^{1} b l(b) d G(b)}{\int_{0}^{1} l(b) d G(b)}$. With a slight abuse of notation, the probability an individual without the virus becomes infected conditional on going outside is $f\left(z, \int_{0}^{1} l(b) \mathrm{d} G(b)\right)$, where $f$ is a continuous function increasing in each argument. Notice that $f$ depends both on the fraction of infected individuals as well as the size of the population that is outside. We allow for this generality as the likelihood of being infected depends not only on the infection rate but also on the likelihood of coming into contact with another person (which increases in the number of people outside). We make no other assumptions on $f$. As before, the expected cost associated with becoming infected is $c>0$.

Total societal value can thus be expressed as

$$
\begin{aligned}
S(z, l)= & \phi\left(\int_{0}^{1} l(b) \mathrm{d} G(b)\right)-\kappa\left(z, \int_{0}^{1} l(b) \mathrm{d} G(b)\right) \\
& -c \int_{0}^{1}(1-b) l(b) f\left(z, \int_{0}^{1} l(b) \mathrm{d} G(b)\right) \mathrm{d} G(b) .
\end{aligned}
$$

Therefore, to maximize social value, a social planner would choose a going out rule $l$ and an infection rate among the working population to solve:

$$
\begin{aligned}
& \max _{z, l} S(z, l) \\
& \text { s.t. } z=\frac{1}{\int_{0}^{1} l(b) \mathrm{d} G(b)} \int_{0}^{1} b l(b) \mathrm{d} G(b) .
\end{aligned}
$$

We now demonstrate that one can suppress the dependence of $f(\cdot, \cdot)$ on the mass of agents working. This allows us to reduce the social planner's problem to selecting an infection rate $z$ in the working population. Proposition 5.1 below shows that conditional on the optimal $z^{*}$, the set of individuals that work is uniquely pinned down. As a result, it is not necessary to explicitly express the dependence of the probability of infection on the size of the workforce.

Proposition 5.1 Suppose $\left(z^{*}, l^{*}\right)$ is the solution to $(S P)$. Then:

1. $z^{*} \leq \pi_{I} \Longrightarrow l^{*}(b)=\left\{\begin{array}{cc}1 & \text { if } b \leq b^{*} \\ 0, & \text { if } b>b^{*},\end{array} \quad\right.$ where $z^{*}=\frac{1}{G(b)} \int_{0}^{b^{*}} b d G(b)$. In words, when $z^{*} \leq \pi_{I}$, it is optimal to only allow types $\left[0, b^{*}\right]$ to go out. 
2. $z^{*}>\pi_{I} \Longrightarrow l^{*}(b)=\left\{\begin{array}{ll}0 & \text { if } b<b^{*} \\ 1, & \text { if } b \geq b^{*},\end{array} \quad\right.$ where $z^{*}=\frac{1}{1-G(b)} \int_{b^{*}}^{1} b d G(b)$.

In words, when $z^{*}>\pi_{I}$, it is optimal to only allow types $\left[b^{*}, 1\right]$ to go out.

Proposition 5.1 demonstrates that if $z^{*}$ is the infection rate given by the solution to (SP), then, at that infection rate it is optimal to induce the maximum feasible subset of the population to go out. To provide intuition, suppose a policy $P$ induces a target infection rate $z^{*}$, but the corresponding "going-out" function $l(\cdot)$ has fewer people going out than the maximum possible. This is suboptimal as there would be a different policy $P^{\prime}$ that is consistent with and induces a lower target infection rate $z^{\prime}<z^{*}$ while also having a total of $\int_{0}^{1} l(b) d G(b)$ workers going out.

An immediate consequence of Proposition 5.1 is the following corollary.

Corollary 5.2 Given any $z^{*}$, define $l^{*}\left(\cdot, z^{*}\right)$ according to Proposition 5.1. Consider the function $Y\left(z^{*}\right)=S\left(z^{*}, l\left(\cdot, z^{*}\right)\right)$. That is, $Y\left(z^{*}\right)$ is the welfare when the planner selects a target infection rate $z^{*}$ and maximizes the mass of workers going out consistent with that $z^{*}$. Then,

$$
\max _{z^{*}} Y\left(z^{*}\right)=\max _{z, l} S(z, l)
$$

It follows that the social planner's problem can be reduced to choosing the optimal infection rate.

\subsection{Model assumptions}

Our conclusions about whom to test and provide transfers rely on an individual's belief about the likelihood of infection being calibrated. Relaxing this assumption will not change the qualitative conclusion that one must deploy both carrots and sticks to align incentives.

We assume that testing is perfect because it makes our point about the need for individuals to sort themselves even stronger. Sorting is even more important when testing is imperfect. It allows one to focus on subgroups who are more likely to be infected so as to reduce the false-positive rate. Section 5.3 explains how to adjust the transfers when testing is imperfect.

Our model incorporates an implicit correlation between expected infection cost and belief (if one has the virus, there is no cost to going outside), but the cost conditional on infection is constant. What if costs are not constant? Individuals who bear a high cost associated with infection are naturally deterred from going out. If one believes that the portion of the population with costs less than the mean is large, the additional transfers needed would be small. However, if the portion of the working population with costs in excess of the mean is large, additional transfers are unavoidable.

If we assume agents are risk-averse rather than risk-neutral, this will have two effects. First, the transfers needed to induce agents to work or stay home will be larger than otherwise. Second, a risk-averse agent may be more reluctant to acquire information about their infection states. Hence, it may no longer be optimal to simply furlough workers if they test positive. 


\subsection{Implementation}

In this section, we briefly discuss the question of implementation feasibility. First, testing at work in a pandemic will not be a one-time affair but conducted at regular intervals. This means the wages and transfers need to scale with the interval between tests. The frequency of testing need not be uniform across individuals in the same workplace nor across workplaces. For example, in a university setting, students with roommates living in high-density dorms would be tested at greater frequency than faculty who are on campus only intermittently. Indeed, many universities tested students frequently throughout the year. Regarding testing at home, some cities have either piloted or implemented door-step testing for the virus. It can also be interpreted as drive-through testing at hospitals or parking lots. Moreover, the results of the test could be shared with employers. What might the cost of such testing be? Levitt et al. (2020) estimate the cost of frequent testing for everyone along with monetary incentives to encourage individuals to get tested at around 80 billion dollars. ${ }^{13}$ Augenblick et al. (2020) also illustrate how frequent testing can be done in a cost-effective manner. Thus, implementing our suggested incentive scheme repeatedly over time is not as costly as one might have thought. ${ }^{14}$

To make our point, we assumed that testing was perfect, but our model easily accommodates imperfect testing. It would change the magnitude of the transfers. False positives, for example, would require one to raise $t_{W}^{+}$. Furthermore, transfers can be implemented as direct payments to agents or as subsidies to employers to keep infected agents asked to stay home on the payroll.

In a dynamic setting, the beliefs of agents about their chance of being infected will change over time. The beliefs of agents who stay home will decline over time. Either they do not fall ill, fall ill and recover, or exit the model due to death. The beliefs of agents who are out may shift upwards over time. The net effect of these two changes will depend on which one is larger. If the second effect is larger, one may wish to pursue a more 'aggressive' policy in the present (i.e. choose a value of $z$ in the present that is lower than what a single period efficiency calculation might dictate). Hence, a dynamic policy could be aggressive initially, becoming more permissive over time, or the reverse. Furthermore, a moral hazard issue can arise in the dynamic setting. If agents recognize that such a scheme of targeted testing and incentives is to be implemented, their behavior will change. For example, when transfers to stay at home are large, it could create a perverse incentive for individuals to intentionally expose themselves in an earlier period in hopes of becoming infected.

\footnotetext{
13 https://www.usatoday.com/story/opinion/2020/04/30/coronavirus-tests-quarantines-incentives-canmake-it-work-column/3048508001/.

14 Especially when compared to the counterfactual of a lockdown with little economic activity.
} 


\section{A Appendix}

\section{A.1 Proofs of main propositions}

Lemma 4.2 In any minimum-cost policy, we must have that $t_{W}^{+}=0$.

Proof Recall that:

$$
\begin{aligned}
& c f\left(z^{*}\right)+r_{W}\left(t_{W}^{+}-t_{W}^{-}\right) \leq 0, \\
& b^{*}=\frac{c f\left(z^{*}\right)+t_{H}^{\neg}-\left(1-r_{W}\right) t_{W}^{-}-r_{W} t_{W}^{-}}{c f\left(z^{*}\right)+r_{W}\left(t_{W}^{+}-t_{W}^{-}\right)} .
\end{aligned}
$$

Suppose otherwise and so $t_{W}^{+}>0$. If $t_{H}^{\neg}>0$, we can decrease both while maintaining the same $b^{*}$, thereby reducing the cost of the policy to the planner. If instead $t_{H}^{\vec{H}}=0$, such a policy incentivizes everyone to go out to work, which contradicts our supposition that $z^{*}<\pi_{I}$.

Proposition 4.3 Suppose testing is only conducted at work $\left(r_{H}=0\right)$. Conditional on the testing probability $r_{W}$, the minimum-cost transfers are as follows:

1. If $c f\left(z^{*}\right)-r_{W} w<0$, the optimal transfer policy has $t_{H}^{\neg}>0, t_{W}^{-}=w$, and $t_{W}^{\neg}=w:$ a transfer to those that stay home, and no transfers in excess of the wage to those that go to work and either test negative or are untested.

2. If $c f\left(z^{*}\right)-r_{W} w>0$, the optimal transfer policy has $t_{H}^{\overrightarrow{ }}>0, t_{W}^{-}>w$, and $t_{W}^{\neg}=w:$ a transfer to those that stay home, and a transfer in excess of the wage to those that work and test negative. Those untested at work receive only their wage.

Proof Case 1: $c f\left(z^{*}\right)-r_{W} w<0$

As we are looking for the minimum cost way of implementing an infection rate $z^{*}$, it must be the case that $t_{W}^{-}=w$ and $t_{W}^{\neg}=w$. Therefore,

$$
\begin{aligned}
b^{*}= & \frac{c f\left(z^{*}\right)+t_{H}^{\neg}-w}{c f\left(z^{*}\right)-r_{W} w}, \\
& \Longrightarrow t_{H}^{\neg}=\left(1-b^{*} r_{W}\right) w-\left(1-b^{*}\right) c f\left(z^{*}\right) \geq 0 .
\end{aligned}
$$

Hence, we must pay those who choose to stay home. The total cost net of wages is

$$
M\left(r_{W} G\left(b^{*}\right)\right)+\left(1-G\left(b^{*}\right)\right)\left[\left(1-b^{*} r_{W}\right) w-\left(1-b^{*}\right) c f\left(z^{*}\right)\right] .
$$

Case 2: $c f\left(z^{*}\right)-r_{W} w>0$

In this case, we require $t_{W}^{-}>w$. In words, we must pay a premium above the wage to those who choose to go out. In the limit, the solution is: $t_{W}^{-}=\frac{c f\left(z^{*}\right)}{r_{W}}>w, t_{W}^{\neg}=w$, 
and $t_{H}^{\neg}=\left(1-r_{W}\right) w$. The total cost net of wages is:

$$
\begin{aligned}
& M\left(r_{W} G\left(b^{*}\right)\right)+\left(1-r_{W}\right)\left(1-G\left(b^{*}\right)\right) w+G\left(b^{*}\right)\left(1-z^{*}\right) r_{W}\left(\frac{c f\left(z^{*}\right)}{r_{W}}-w\right), \\
& =M\left(r_{W} G\left(b^{*}\right)\right)+\left(\left(1-r_{W}\right)\left(1-G\left(b^{*}\right)\right)-G\left(b^{*}\right)(1-z) r_{W}\right) w \\
& \quad+G\left(b^{*}\right)\left(1-z^{*}\right) c f\left(z^{*}\right), \\
& =M\left(r_{W} G\left(b^{*}\right)\right)+\left(1-r_{W}+G\left(b^{*}\right) z^{*} r_{W}\right) w-G\left(b^{*}\right) w+G\left(b^{*}\right)\left(1-z^{*}\right) c f\left(z^{*}\right) .
\end{aligned}
$$

Lemma 4.4 In any minimum-cost policy, we must have that $t_{H}^{-}=0$.

Proof Recall that the policy must satisfy

$$
\begin{aligned}
& c f\left(z^{*}\right)+r_{H}\left(t_{H}^{-}-t_{H}^{+}\right) \leq 0, \\
& b^{*}=\frac{c f\left(z^{*}\right)+\left(1-r_{H}\right) t_{H}^{\neg}-t_{W}^{\neg}+r_{H} t_{H}^{-}}{c f\left(z^{*}\right)+r_{H}\left(t_{H}^{-}-t_{H}^{+}\right)} .
\end{aligned}
$$

Suppose for the sake of contradiction that $t_{H}^{-}>0$. If $t_{H}^{\neg}>0$, we can decrease both, reducing the cost of the policy, while maintaining the same $b^{*}$. Suppose instead that $t_{H}^{\neg}=0$. Then a policy that satisfies the inequalities above must be such that no one goes to work, which is a contradiction.

Proposition 4.5 Suppose testing is only conducted at home $\left(r_{W}=0\right)$. Conditional on the testing probability $r_{H}$, the minimum-cost transfers are as follows:

1. If cf $\left(z^{*}\right)-w>0$, the optimal transfer policy has $t_{H}^{\overrightarrow{ }}=0, t_{H}^{+}>0$, and $t_{W}^{\overrightarrow{ }}>w$ : a transfer to those who stay home and test positive as well as a transfer in excess of the wage to those who go to work.

2. If $c f\left(z^{*}\right)-w<0$, the optimal transfer policy has $t_{H}^{\overrightarrow{ }}>0, t_{H}^{+}>0$, and $t_{W}^{\overrightarrow{ }}=w$ : a transfer to those that stay home and either test positive or do not get tested, and no transfer in excess of the wage to those who go to work.

Proof As before, we have two cases to consider.

Case 1: $c f\left(z^{*}\right)-w>0$

Clearly, in an optimal policy we must have $t_{H}^{\neg}=0$. Thus, $t_{W}^{\neg}=c f\left(z^{*}\right)$ and $t_{H}^{+}=$ $\frac{c f\left(z^{*}\right)}{r_{H}}$. Total cost net of wages will be

$$
\begin{aligned}
& M\left(r_{H}\left(1-G\left(b^{*}\right)\right)\right)+G\left(b^{*}\right)\left(c f\left(z^{*}\right)-w\right)+r_{H}\left(\int b(1-l(b)) \mathrm{d} G(b)\right) \frac{c f\left(z^{*}\right)}{r_{H}} \\
& \quad=M\left(r_{H}\left(1-G\left(b^{*}\right)\right)\right)+G\left(b^{*}\right)\left(c f\left(z^{*}\right)-w\right)+\left(\pi_{I}-G\left(b^{*}\right) z\right) c f\left(z^{*}\right) \\
& \quad=M\left(r_{H}\left(1-G\left(b^{*}\right)\right)\right)+G\left(b^{*}\right)(1-z) c f\left(z^{*}\right)-G\left(b^{*}\right) w+\pi_{I} c f\left(z^{*}\right) .
\end{aligned}
$$


Notice that the home testing probability does not affect the total cost of the transfers net of wages.

Case 2: $c f\left(z^{*}\right)-w<0$

It must be the case that $t_{H}^{-}=0$ and $t_{W}^{\neg}=w$. Therefore,

$$
b^{*}=\frac{c f\left(z^{*}\right)+\left(1-r_{H}\right) t_{H}^{\neg}-w}{f\left(z^{*}\right) c-r_{H} t_{H}^{+}} .
$$

Suppose we fix $t_{H}^{+}>0$ and $t_{H}^{\neg} \geq 0$ such that the above expression holds. Consider the costs associated with the "stay at home" transfers:

$$
\left(1-r_{H}\right) t_{H}^{\neg}\left(1-G\left(b^{*}\right)\right)+r_{H} t_{H}^{+} \int_{b^{*}}^{1} b d G(b)
$$

As the expression for $b^{*}$ must hold, we can see that

$$
\frac{\partial\left(1-r_{H}\right) t_{H}^{\neg}}{\partial r_{H} t_{H}^{+}}=-b^{*} .
$$

Thus, the optimal policy has $t_{H}^{+}=\frac{c f\left(z^{*}\right)}{r_{H}}$ and $t_{H}^{\neg}=\frac{w-c f\left(z^{*}\right)}{1-r_{H}}$. Total cost is:

$$
\begin{aligned}
& M\left(r_{H}\left(1-G\left(b^{*}\right)\right)\right)+\left(w-c f\left(z^{*}\right)\right)\left(1-G\left(b^{*}\right)\right)+c f\left(z^{*}\right) \int_{b^{*}}^{1} b d G(b) \\
& \quad=M\left(r_{H}\left(1-G\left(b^{*}\right)\right)\right)+\left(w-c f\left(z^{*}\right)\right)\left(1-G\left(b^{*}\right)\right)+c f\left(z^{*}\right)\left(\pi_{I}-z^{*} G\left(b^{*}\right)\right), \\
& \quad=M\left(r_{H}\left(1-G\left(b^{*}\right)\right)\right)+c f\left(z^{*}\right)\left(w-c f\left(z^{*}\right)\right)\left(1-G\left(b^{*}\right)\right)+c f\left(z^{*}\right)\left(\pi_{I}-z^{*} G\left(b^{*}\right)\right), \\
& \quad=M\left(r_{H}\left(1-G\left(b^{*}\right)\right)\right)+G\left(b^{*}\right)\left(1-z^{*}\right) c f\left(z^{*}\right)-G\left(b^{*}\right) w+\pi_{I} c f\left(z^{*}\right)+w-c f\left(z^{*}\right) .
\end{aligned}
$$

Proposition 4.6 The value of the minimum cost test-at-home policy as a function of $r_{H}$ declines as $r_{H} \rightarrow 0$. However, the stay-at-home transfer conditional on testing positive scales with $\frac{1}{r_{H}}$, that is, $t_{H}^{+}=\frac{c f\left(z^{*}\right)}{r_{H}}$.

Proof From the proof of Proposition 4.5, the term $r_{H}$ is only present in the cost of testing (as an input into the function $M$ ). The total cost of transfers net of wages is independent of $r_{H}$. Hence, the minimum cost is achieved by setting $r_{H}=0$.

Proposition 4.7 Suppose cf $\left(z^{*}\right)-w>0$ :

1. If $\pi_{I} \geq \frac{w}{c f\left(z^{*}\right)}$, then the minimum-cost test-at-work policy that induces infection rate $z^{*}$ is less costly than any test-at-home policy that induces infection rate $z^{*}$.

2. If $\pi_{I}<\frac{w}{c f\left(z^{*}\right)}$ and testing is sufficiently costly, there is a test-at-home policy that induces infection rate $z^{*}$ and is less costly than the minimum-cost test-at-work policy that induces infection rate $z^{*}$. 
Proof Let $C_{W}(r)$ denote the cost of the testing at work policy when tests are conducted with probability $r$. The minimum cost policy has $\operatorname{cost} C_{W}^{*}$, which is achieved at $r^{*}$.

When testing at home only, the minimum cost is:

$$
C_{H}=\pi_{I} c f\left(z^{*}\right)+G\left(b^{*}\right)\left(1-z^{*}\right) c f\left(z^{*}\right)-w G\left(b^{*}\right) .
$$

$$
\text { If } \frac{w}{c f\left(z^{*}\right)} \leq \pi_{I} \Longrightarrow\left(1-G\left(b^{*}\right)\right) w \leq-G\left(b^{*}\right) w+\pi_{I} c f\left(z^{*}\right)
$$

$$
\Longrightarrow C_{W}(0) \leq C_{H}
$$

Since $C_{W}^{*} \leq C_{W}(0)$, the first part of the proposition follows.

For the second part of the proposition, we compare $C_{H}$ and $C_{W}^{*}$ directly:

$$
C_{H} \leq C_{W}^{*} \Longleftrightarrow \pi_{I} \leq \frac{M\left(r^{*} G\left(b^{*}\right)\right)}{c f\left(z^{*}\right)}+\left(1-r_{W}^{*}+G\left(b^{*}\right) z^{*} r_{W}^{*}\right) \frac{w}{c f\left(z^{*}\right)} .
$$

If $\pi_{I}<\frac{w}{c f\left(z^{*}\right)}$, then there exists $k \in(0,1)$ such that $\pi_{I}=k \cdot \frac{w}{c f\left(z^{*}\right)}$. It follows then that a sufficient condition for $C_{H} \leq C_{W}^{*}$ is $r_{W}^{*}<\frac{1-k}{1-G\left(b^{*}\right) z^{*}}$. This occurs when testing is sufficiently costly:

$$
M^{\prime}\left(\frac{(1-k) G\left(b^{*}\right)}{1-G\left(b^{*}\right) z^{*}}\right) \geq \frac{\left(1-G\left(b^{*}\right) z^{*}\right) w}{G\left(b^{*}\right)} .
$$

Remark Note that when testing is costless, $r_{W}^{*}=1$ and $M(\cdot)=0$, which implies:

$$
C_{H} \leq C_{W}^{*} \Longleftrightarrow \pi_{I} \leq G\left(b^{*}\right) z \frac{w}{c f\left(z^{*}\right)}
$$

\section{A.2 Social planner problem: microfoundation}

Lemma A.1 Fix $z^{*}$. If $z^{*} \leq \pi_{I}$, define $l^{*}\left(b, z^{*}\right)=\left\{\begin{array}{ll}0 & b>b^{*}, \\ 1 & b \leq b^{*}\end{array}\right.$ where $b^{*}=$ $\frac{\int_{0}^{b^{*}} b d G(b)}{G\left(b^{*}\right)}=z^{*}$. If $z^{*}>\pi_{I}$, define $l^{*}\left(b, z^{*}\right)=\left\{\begin{array}{ll}1 & b \geq b^{*}, \\ 0 & b<b^{*}\end{array}\right.$ where $b^{*}=\frac{\int_{b^{*}}^{1} b d G(b)}{1-G\left(b^{*}\right)}=$ $z^{*}$.

The function $l^{*}$ solves:

$$
\begin{aligned}
& \max _{l(\cdot) \in[0,1]} \int_{0}^{1} l(b) d G(b), \\
\text { s.t. } & \frac{\int_{0}^{1} b l(b) d G(b)}{\int_{0}^{1} l(b) d G(b)} \leq z^{*} .
\end{aligned}
$$


Proof Observe that the constraint can be rewritten as a linear constraint by moving the denominator to the right-hand side and rearranging. The observation now follows from a straightforward Lagrangean relaxation.

The function $l^{*}\left(\cdot, z^{*}\right)$ describes the maximal number of people that can go out under a consistent policy that induces infection rate $z^{*}$.

Proposition 5.1 Suppose $\left(z^{*}, l^{*}\right)$ is the solution to (SP). Then:

1. $z^{*} \leq \pi_{I} \Longrightarrow l^{*}(b)=\left\{\begin{array}{cc}1 & \text { if } b \leq b^{*} \\ 0, & \text { if } b>b^{*},\end{array} \quad\right.$ where $z^{*}=\frac{1}{G(b)} \int_{0}^{b^{*}} b d G(b)$.

In words, when $z^{*} \leq \pi_{I}$, it is optimal to only allow types $\left[0, b^{*}\right]$ to go out.

2. $z^{*}>\pi_{I} \Longrightarrow l^{*}(b)=\left\{\begin{array}{ll}0 & \text { if } b<b^{*} \\ 1, & \text { if } b \geq b^{*},\end{array} \quad\right.$ where $z^{*}=\frac{1}{1-G(b)} \int_{b^{*}}^{1} b d G(b)$.

In words, when $z^{*}>\pi_{I}$, it is optimal to only allow types $\left[b^{*}, 1\right]$ to go out.

Proof We start by proving the first statement. Suppose a policy $P$ induces a target infection rate $z^{*}$ and the planner selects a corresponding going-out function $\hat{l}(\cdot)$, where $\int_{0}^{1} \hat{l}(b) d G(b)<\int_{0}^{1} l^{*}\left(b, z^{*}\right) d G(b)$. Since $\int_{0}^{1} l^{*}\left(b, z^{*}\right) d G(b)$ is monotonically decreasing in $z^{*}$ and $\lim _{z^{*} \rightarrow 0} \int_{0}^{1} l^{*}\left(b, z^{*}\right) d G(b)=0$, there exists $z^{\prime}<z^{*}$ such that $\int_{0}^{1} l^{*}\left(b, z^{\prime}\right) d G(b)=\int_{0}^{1} \hat{l}(b) d G(b)$. However, this means that $S\left(z^{*}, \hat{l}(\cdot)\right)<$ $S\left(z^{\prime}, l^{*}\left(\cdot, z^{\prime}\right)\right)$. Thus, a target infection rate of $z^{*}$ with going-out function $\hat{l}(\cdot)$ can not be optimal.

The second statement follows from combining the first with Lemma A.1.

\section{References}

Acemoglu D, Chernozhukov V, Werning I, Whinston M (2020) A multi-risk model with optimally targeted lockdown. forthcoming, American Economic Review: Insights

Akbarpour M, Cook C, Marzuoli A, Mongey S, Nagaraj A, Saccarola M, Tebaldi P, Vasserman S, Yang H (2020) Socioeconomic network heterogeneity and pandemic policy response. Working Paper

Alvarez FE, Argente D, Lippi F (2020) A simple planning problem for COVID-19 lockdown. forthcoming, American Economic Review: Insights

Armbruster B, Brandeau ML (2007) Contact tracing to control infectious disease: when enough is enough. Health Care Manag Sci 10:341-355

Atkeson A (2020) How deadly is COVID-19? Understanding the difficulties with estimation of its fatality rate. Working Paper 26965, National Bureau of Economic Research

Augenblick N, Kolstad J, Obermeyer Z, Wang A (2020) Group testing in a pandemic: the role of frequent testing, correlated risk, and machine learning. Working Paper

Ben-Porath E, Dekel E, Lipman BL (2014) Optimal allocation with costly verification. Am Econ Rev 104(12):3779-3813

Bienstock D, Zenteno AC (2015) Models for managing the impact of an epidemic. arXiv preprint

Bobkova N, Chen Y, Eraslan H (2021) Optimal group testing with heterogeneous risks. Working Paper

Bueno de Mesquita E, Shadmehr M (2020) Coordination and social distancing: inertia in the aggregate response to COVID-19. Becker Friedman Institute for Economics Working Paper No. 2020-53

Carnehl C, Fukuda S, Kos N (2021)Epidemics with behavior. Working Paper

Dasaratha K (2020) Virus dynamics with behavioral responses. Working Paper

Dorfman R (1943) The detection of defective members of large populations. Ann Math Stat 14:436-440

Du D-Z, Hwang FK (1992) Combinatorial group testing and its applications. World Scientific, Singapore

Eichenbaum MS, Rebelo S, Trabandt M (2020) The macroeconomics of epidemics. Working Paper 26882, National Bureau of Economic Research 
Eichenbaum M, Rebelo S, Trabandt M (2020) The macroeconomics of testing during epidemics. mimeo Ely J, Galeotti A, Jann O, Steiner J Optimal test allocation. J Econ Theory, forthcoming

Engle S, Keppo J, Kudlyak M, Quercioli E, Smith L, Wilson A (2021) The behavioral SIR model, with applications to the Swine Flu and COVID-19 pandemics. Working Paper

Fang H, Wang L, Yang Y (2020) Human mobility restrictions and the spread of the novel coronavirus (2019-nCoV) in China. J Public Econ 191

Farboodi M, Jarosch G, Shimer R (2020) Internal and external effects of social distancing in a pandemic. Becker Friedman Institute for Economics Working Paper 2020-47

Favero CA, Ichino A, Rustichini A (2020) Restarting the economy while saving lives under COVID-19. Working Paper

Gadarian SK, Goodman SW, Pepinsky TB (2021) Partisanship, health behavior, and policy attitudes in the early stages of the COVID-19 pandemic. PLoS ONE 16(4):e0249596

Glover A, Heathcote J, Krueger D, Rios-Rull JV (2020) Health versus wealth: on the distributional effects of controlling a pandemic. Working Paper 27046, National Bureau of Economic Research

Gollier C (2020) Optimal group testing to exit the COVID confinement. Tolouse School Econ

Gollier C, Gossner O (2020) Group testing against covid-19. Covid Econ 1(2):32-42

Kruse T, Strack P (2020) Optimal control of an epidemic through social distancing. Cowles Foundation Working Paper

Levitt S, Romer P, Severts J (2020) Pandemillions: how to get healthy people to repeatedly test for COVID-19

Li Y (2020) Mechanism design with costly verification and limited punishments. J Econ Theory 186

Lipnowski E, Ravid D (2021) Group testing for quarantine decisions. J Econ Theory, forthcoming

Mas-Colell A, Whinston MD, Green JR (1995) Microeconomic theory. Oxford University Press, New York

McAdams D (2020) Nash SIR: an economic-epidemiological model of strategic behavior during a viral epidemic. Covid Econ 1:115133

Preciado V, Enyioha C, Jadbabaie A, Pappas GJ, Zargham M (2014) Optimal resource allocation for network protection against spreading processes. IEEE Trans Control Netw Syst 1(1):99-108

Publisher's Note Springer Nature remains neutral with regard to jurisdictional claims in published maps and institutional affiliations. 